\title{
Use of projector based augmented reality to improve manual spot-welding precision and accuracy for automotive manufacturing
}

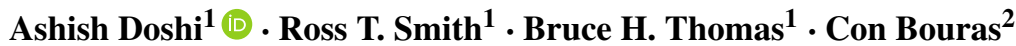

Received: 18 March 2016 / Accepted: 11 July 2016 / Published online: 25 July 2016

(C) The Author(s) 2016. This article is published with open access at Springerlink.com

\begin{abstract}
This paper presents the use of a projector-based spatial augmented reality system in an industrial quality assurance setting to highlight spot-weld locations on vehicle panels for manual welding operators. The aim of this work is to improve the precision and accuracy of manual spot-weld placements with the aid of visual cues as a proactive step by the automotive manufacturer to enhance product quality. The prototype system was deployed at General Motors (GM) Holden plant in Elizabeth, Australia on the production line building Holden Cruze vehicles. Production trials were conducted and techniques developed to analyse and validate the precision and accuracy of spot-welds both with and without the visual cues. A reduction of $52 \%$ of the standard deviation of manual spot-weld placement was observed when using augmented reality visual cues. The average standard deviation with-AR assistance (19 panels and 114 spot-welds) was calculated at $1.94 \mathrm{~mm}$ compared to without-AR (45 panels and 270 spot-welds) at $4.08 \mathrm{~mm}$. All welds were within the required specification and panels evaluated in this study were used as the final product made available to consumers. The visual cues enabled operators to spot-weld at a higher degree of precision and accuracy.
\end{abstract}

Grant ID: AutoCRC C1-53 AR Projection System for Work Cells 2

Ashish Doshi

ashishd@ieee.org

1 Wearable Computer Laboratory, University of South Australia, Mawson Lakes Campus, Adelaide, Australia

2 GM Holden Vehicle Operations, Elizabeth, South Australia, Australia
Keywords Automotive manufacturing - Spot-weld validation - Spatial augmented reality $\cdot$ Visualisation

\section{Introduction}

General Motors (GM) Holden have a long history of producing safe and reliable road vehicles. ${ }^{1}$ As per their charter, they are actively seeking to improve existing manufacturing practices and vehicle designs using new technology to meet the challenges of future automotive requirements. This is an evolving process that the automotive industry as a whole are involved in since the Model T assembly line ${ }^{2}$ was revolutionised in 1913 to gain a production advantage in the automotive market. This work on integrating augmented reality technology on the production line for manual spotwelding was conducted in collaboration with GM Holden Vehicle Operations at their facility in Elizabeth, Australia.

The vehicle body assembly plant in Elizabeth, Australia opened in 1962 and has continuously undergone revisions and modernisations to achieve production efficiency. Due to the expense of robotic dexterity, not all of the assembly processes have been automated. This meant spot-welding and adhesive applications of small assembly panels are performed manually. There are two types of testing conducted on the production line. Destructive testing is performed periodically by taking a completed vehicle body out of production [1]. Non-destructive testing of weld quality assurance is performed more frequently by the use of a single probe ultrasonic tester to measure the thickness of the spot-weld [2]. Visual inspection checks are undertaken to ascertain whether spot-welds are in the appropriate location

\footnotetext{
${ }^{1}$ http://www.holden.com.au/about/innovation/safety.

${ }^{2}$ http://corporate.ford.com/company/history.html.
} 
and to ensure that the overall pattern conforms to the work instruction [3]. In the absence of suitable stencils or physical guides, visual inspection is a viable method.

Existing manual production stations rely on job specific instructions which operators commit to memory. Physical guides are placed on the welding part to help welders locate a subset of the required welds (these are mainly for stud-welds), but due to size restrictions there are no physical guides for every weld. Therefore, quality assurance inspectors are limited to prioritising critical stud-welds, testing of spot-weld thickness, and visual inspection of weld placements.

The engineering requirements in a production environment is such that the developed system has to work alongside existing plant infrastructure, no interference with production cycle (expensive if there was any), operators should not wear or use additional wearable device without specific approval and any prototype has to adhere to plant safety regulations. Unlike the vision-based monitoring system introduced by [4] which was limited to detection and evaluation of spot-welds, the goal of our work is to explore and investigate the use of augmented reality in the production environment. Our solution is to develop a projector based Spatial Augmented Reality (SAR) system that can be integrated at production stations as required and works alongside existing plant infrastructure. The projection system becomes an active part of the welding process, i.e. specifying spot-weld placements. SAR superimposes computer generated virtual imagery (e.g. virtual cues) directly onto the physical objects' surfaces. The virtual cues act as visual aids to help the operators weld in the correct location on the panel. The system is unobtrusive to the welders and they do not require to hold or wear additional equipment or personal protective equipment (PPE), which would interfere with their spot-welding tasks.

The SAR system will not only enhance the operator's experience but also aid and improve the manufacturing process as a whole, without hindering the user. Unlike [5] that proposed to setup a virtual welder trainer in a training centre, the SAR visualisation system can also be used for on-the-job training of operators with higher precision and accuracy. This investigation details an industrial proof-ofconcept of the SAR projector system validated on an operational production line, which is safe and reliable within the manufacturing environment [6].

This paper is organised as follows. Section 2 describes the prior work of using augmented reality (AR) and visualisation methods in the manufacturing industry. Section 3 details existing processes within the plant whilst Section 4 describes the production line and challenges faced when commissioned as the test bed for the SAR visualisation system. The design of suitable virtual cues is presented in Section 5. The SAR prototype system was developed and installed on the production line at the GM Holden plant in Elizabeth, Australia. An offline pilot study was conducted, and this is presented in Section 6. Section 7 describes the production trials conducted and presents the outcome of the precision and accuracy tests. Discussion of the results is also included in this section. This paper is concluded with our summary and recommendations for further work in this area in Section 8.

\section{Prior work}

A large portion of weld quality research has focussed to date on weld gun technology, such as resistive [7, 8], gas metal arc [9] and laser [10] for manual operators, automated systems as used by manufacturing robots [11] and weld dynamics, such as spot-weld formations [12] and weld heat distribution $[10,13]$. However, not much research have been directed towards weld placements. Echtler et al. [14] did propose an augmented reality-based method to aid in weld placements on the production line. This was subsequently incorporated within the weld gun design.

Augmented reality (AR) is increasingly becoming a popular method for a user to interact with technology either to obtain more information or to enhance their experience for a specific task/application. This is especially true for single user interactions in mobile augmented reality. Automotive industries are slowly but surely adopting AR for user interaction. They are using it for marketing products as well as for Head-Up Displays (HUD) to project information onto vehicle windscreen. BMW [14] and Volkswagen [15] have used AR on their production line, but AR is still an uncommon practice among the wider automotive industry.

The automotive manufacturing industry started integrating new technologies such as AR in the late-1990s. For example, Reiners et al. [16] explored a Head Worn Display (HWD)-based AR system on a doorlock assembly. The user would wear the HWD with transparent glasses. The optical tracker would track the head pose, the camera would track the pose of the door and identify AR markers. The virtual object of the doorlock is thus rendered onto the eyewear with audio instructions on the assembly task. Though the system received positive feedback of its potential, one of its major drawbacks was the optical tracker used caused lag in the tracking phase. This was a major issue as they could not sufficiently compensate for head movements reliably.

Although using HWDs have been a common approach to deploying AR technology for manufacturing, findings reported in [17] show that virtual-to-real space perception can lead to significant assembly inaccuracies. Hence, the requirement of better plant integration systems. The ARVIKA [18] project emphasised a similar conclusion. Over a 4-year period, the project was aimed at fostering 
research and development partnerships between automotive industries and academics in Germany on AR. They observed that though AR research had progressed significantly in an academic environment, the biggest drawback to AR integration in manufacturing was operator usability (for instance interactions and workflow interruptions), safety regulations, and environmental conditions in the plant. Therefore, for a widespread acceptance of AR on the production line, these issues have to be addressed, which is the foundation of our work. This conclusion was further supported by [19] through various AR projects at Daimler Chrysler (in Germany), ranging from servicing and maintenance of vehicles through to prototype design and plant organisation.

Reference [20] along with [21] proposed a desktopbased AR system for manual assembly and planning process. Their systems reposition virtual models over existing plant layout to facilitate easier plant process planning. This helped engineers to visualise production layout changes and optimise the workflow based on production requirements. A similar system was developed in [22] for use on mobile devices.

AIRBUS Military [23] trialled mobile AR work instructions for electrical harness routing on the Airbus Military aircraft frame. Though there was limited testing, the reported user satisfaction was promising and showed that using AR as a virtual aid can significantly improve performance.

Zhou et al. [24] investigated the feasibility of using SAR with laser projectors to display spot weld positions and its information onto a vehicle panel. Though this was successfully tested in a laboratory environment, the system could not be deployed on the production line due to safety concerns [25]. In an open and multi-user production station, the risk associated with the hazard of working with reflective aluminium panels is quite high, hence, the use of such laser projectors was not advisable. Their proposal however was only operative for a single work station and has limited visualisation field of view due to plant physical constraints. Manufacturers such as BMW [14] and Volkswagen [15] have trialled an in-situ welding facilitator using laser projection. Their system is based on AR markers [26], where markers are placed at specific positions on the assembly line to help with position and orientation identification thereby offsetting it to place the virtual graphic in the correct location. Two ways have been proposed to do this. One is with a specialised welding gun [14] and the other is with laser projectors [24, 27]. In both the cases, the AR interaction is limited to single user, is quite expensive and can pose as a safety hazard in the plant. In contrast, our system addresses the current assembly AR limitations, with multi-user interaction, full projective coverage of the object/panel surface, increase of manual production efficiency (accuracy and precision) and to minimize unnecessary burden on operators when integrating new AR technology [28].

\section{The production line}

This section describes the existing process in the GM Holden plant in Elizabeth, South Australia, Australia and motivation behind the development of the SAR prototype for the production line. The production station that was chosen is one among many manual work stations of the GM Holden Cruze production pipeline. The chosen station was identified as having a large work-area for ease of SAR prototype development and consists of a vehicle panel sitting firmly on a static fixture. This provided the ideal surface area to display the AR visualisations. Furthermore, the vehicle panel's positioning could be replicated within the visualisation laboratory for testing purposes.

\subsection{Existing physical environment}

There are two variants of the GM Holden Cruze that spotwelding are performed at this station. The majority of welding locations for these two variants are identical, but there are a number of spot-welds locations that differ. The surface area of the vehicle panel is approximately $1.5 \mathrm{~m}$ (width) and $1.5 \mathrm{~m}$ (length) and is placed on a static fixture at $1 \mathrm{~m}$ above ground. Figure 1 provides a depiction of the space and physical constraints around the fixture.

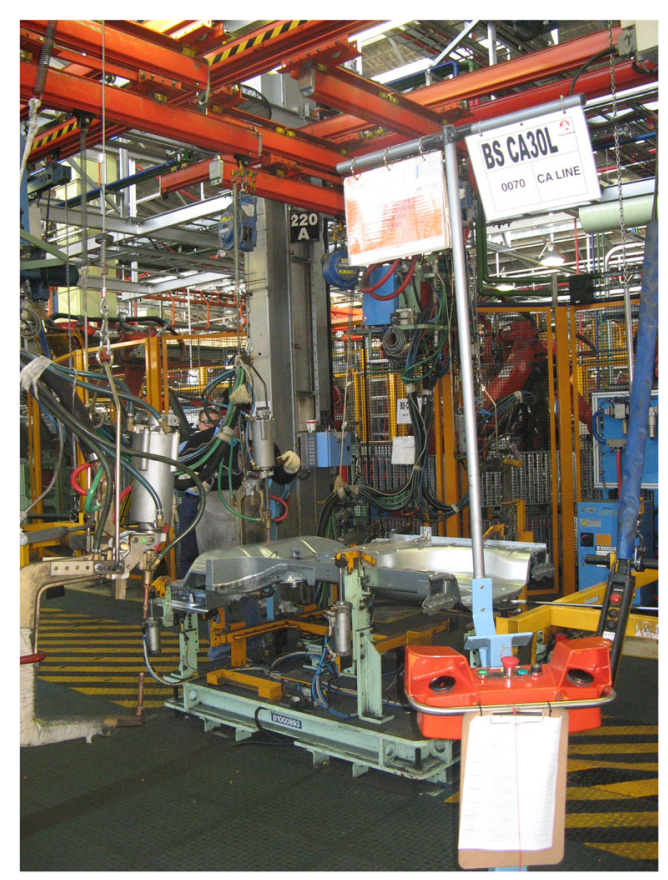

Fig. 1 Manual welding work station at GM Holden Vehicle Operations, Elizabeth, Australia 


\subsection{Workflow}

Two operators are assigned to the station where the welding is carried out. Between them, they weld 62 spot-welds and six stud-welds within the allocated $180 \mathrm{~s}$. The time includes hoisting the panel onto the fixture, securing it, welding, verification of the welds, unfasten the clamps and hoisting the work piece onto a rotatable fixture for moving the panel into the robotic welding station. As per our observations, the workflow for this production station is as follows:

1. If fixture is clear, hoist new panel onto fixture.

2. Press palm button to clamp the fixture.

3. With reference to Fig. 2b, weld guns on the left and front (bottom of layout) are taken.

4. One operator spot-welds on the left-side of the panel and the other on the front.

5. Weld guns are returned to position.

6. With reference to Fig. 2b, weld guns on the right and front right (bottom of layout) are taken.

7. Whilst one operator is handling the stud-weld gun, the other puts the hand-held fixtures for stud-weld positioning in place.

8. One operator spot-welds on the right-side of the panel and the other places stud-welds on the front.

9. Weld guns are returned to position and stencil is removed.

\section{a}
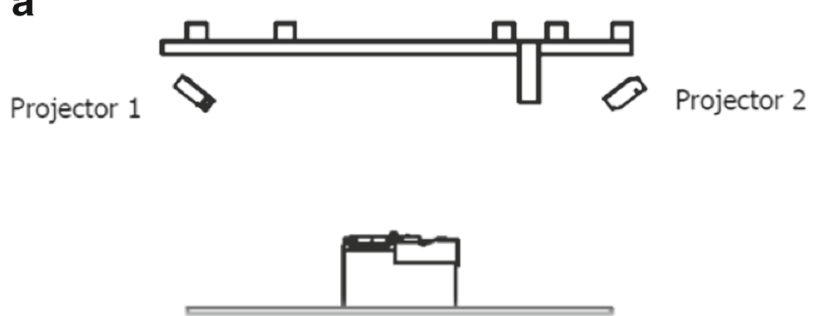

b

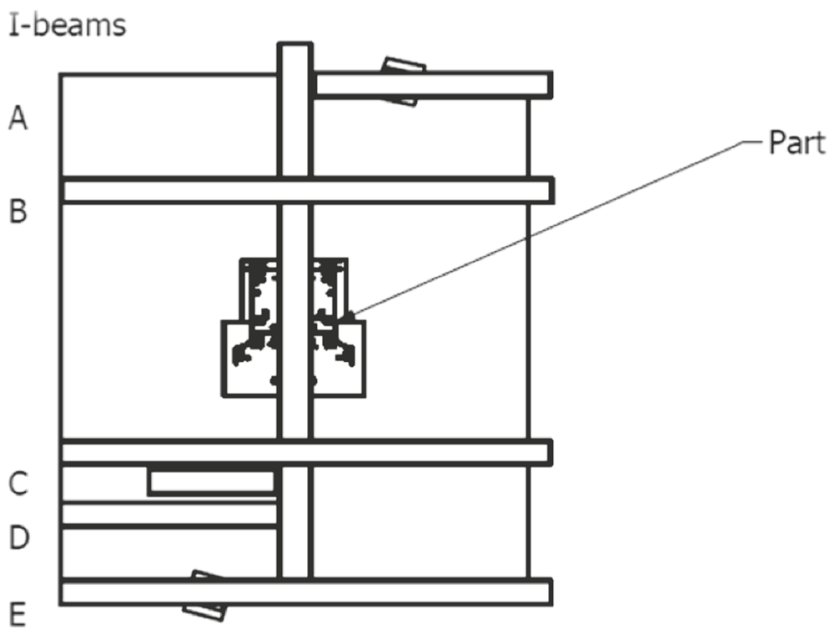

Fig. 2 Proposed schematic with a side view and $\mathbf{b}$ top view
10. The panel is unclamped from the fixture.

11. Hoist completed panel onto rotating fixture for entry into automated welding station.

12. Repeat process.

The observed workflow has been optimised for the operators' time to handle weld guns, perform welding, and quick verification of the weld positions. To manage the quality assurances within the production cycle, inspectors perform ultrasonic thickness test of the spot-welds whilst the operators are welding at other spot-weld positions.

\subsection{Human requirements}

During hoisting of the panel between fixtures, there is a large amount of movement around all the stations with different panels being hoisted into positions at other stations as well, hence, an important issue is to maintain an unobstructed floor area for operators' safety. Although a wearable device such as used in [27] would have a number of advantages, a wearable system was impractical for use on this production line (especially with large surface area of the panel) and could lead to slower production turnover.

\section{SAR enhancements}

Due to the layout of the station and the close proximity to other stations, the placement of projectors could not be mounted from the floor, as this would disrupt the natural foot flow of the operators. Hence, an I-beam mounted solution was considered. The schematic for the layout is presented in Fig. 2.

\subsection{Physical layout}

With reference to Fig. 2, our solution incorporates two projectors in the system. With two operators at this station, two projectors are required to cover for occlusions during welding. A single projector is not adequate to handle occlusions. As can be observed in Fig. 1, the location and positioning of the weld gun could potentially cause occlusions. For example, whilst one is being used on the back of the panel (flat surface area), the weld gun can occlude the projected light at the front (outward vertical bend). Hence, some spotweld positions might be visible to the second operator and some might not, thus interfering with overall workflow. Other occlusion examples are clamping fixtures and human interference. With two projectors, there is at least $80 \%$ overlapping projector field of view coverage between the two projectors. The front end of the panel is curved in and is therefore hidden from one projector. This is compensated for by the second projector, with all of the visible surfaces 
for the panel within the fields of view of the projectors. A third projector directly orthogonal and above the panel would have been preferable, but this was not possible due to overhead monorail for hoisting panels. Although additional projectors were considered, this was not followed through due to the physical constraints of this station layout.

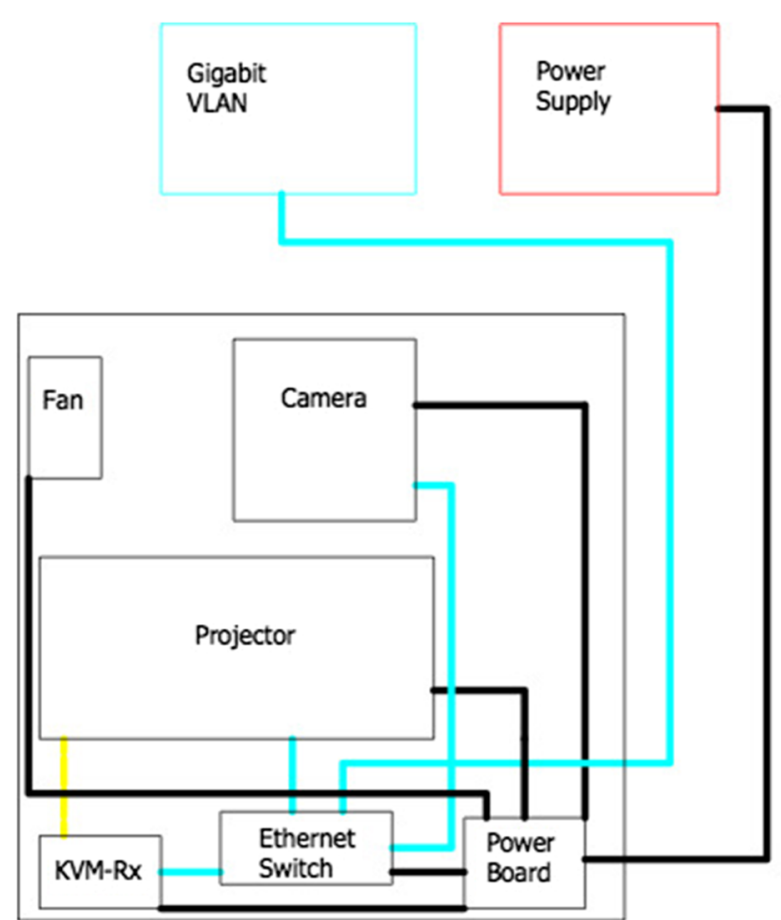

Enclosure 1

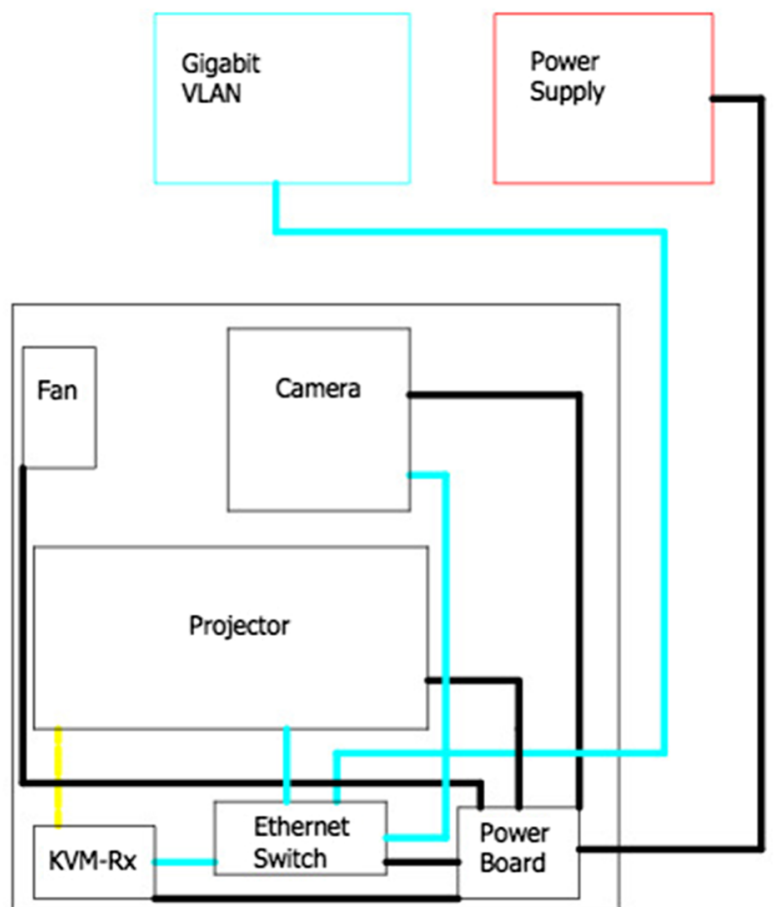

Enclosure 2

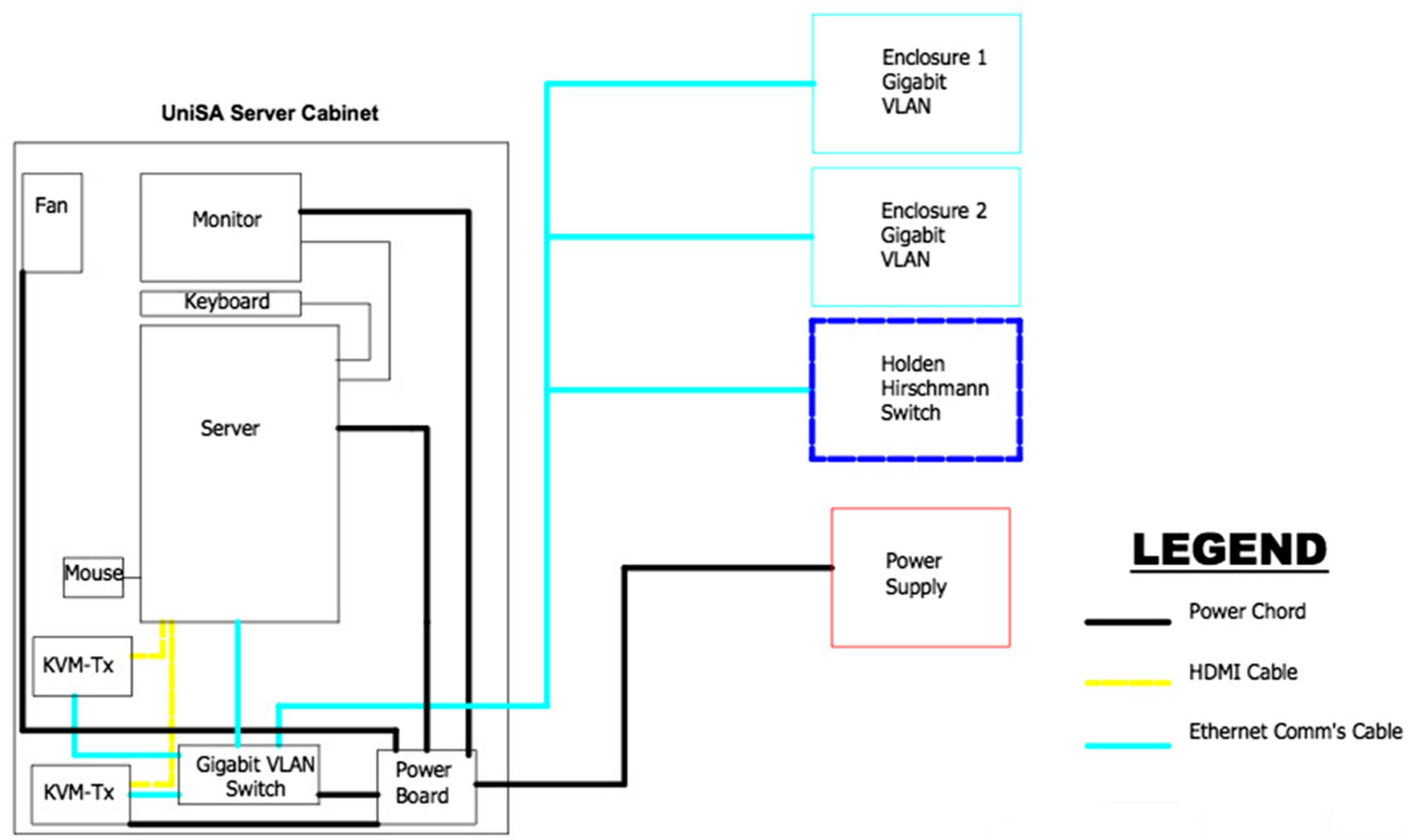

Fig. 3 SAR network diagram 


\subsubsection{Private network}

With the projector layout finalised, the virtual private network for the SAR system was determined. The network schematic is presented in Fig. 3.

There were many challenges faced when developing the SAR prototype. Although deciding which equipment was suitable and the development of the prototype was challenging, integration of the private network alongside existing plant data network was just as demanding. The video signals from the computing system to the projector were transmitted over KVM switches that converted HDMI data stream to network data stream and vice-versa. The system also employed digital IP video cameras that required networking.

The projector bandwidth requirement at $60 \mathrm{~Hz}$ is calculated to be approximately $443 \mathrm{Mbps}$. Similarly, the camera bandwidth requirement of 15 frames per second capture at $60 \mathrm{~Hz}$ is calculated to be approximately $239 \mathrm{Mbps}$.

Hence, the total imaging bandwidth requirement per enclosure is $443 \mathrm{Mbps}+239 \mathrm{Mbps}=682 \mathrm{Mbps}$. Based on the physical layout as per Fig. 2 and the network schematic as in Fig. 3, the cabling requirements were estimated to be $30 \mathrm{~m}$ for enclosure 1 and $35 \mathrm{~m}$ for enclosure 2 . To account for physical plant constraints of laying the cables, the requirement was set at $40 \mathrm{~m}$ and heat resistant as per plant environment. The ethernet cables have to be shielded from electromagnetic interference from the weld guns and alien crosstalk from other electronic devices in the plant. With due consideration to the aforementioned requirements, Cat7a SFTP ethernet cables were chosen. This form of ethernet has a higher bandwidth capacity (up to $1000 \mathrm{MHz}$ ) and a higher signal-to-noise ratio per $100 \mathrm{~m}$ compared to other cables. Due to its copper-shielding jacket and foil screened twisted pair wires, this form of cable significantly reduces alien crosstalk between wires and nearby equipment.

\subsubsection{Prototype hardware}

The SAR system consists of the following components: projectors (NEC NP-PA500UG), cameras, ${ }^{3} \mathrm{KVM},{ }^{4}$ power rails and network switches (HUG-500SE). Since the projectors were to be mounted overhead and the lens of the projectors was approximately $4.3 \mathrm{~m}$ (projector 1 -back) and $4.5 \mathrm{~m}$ (projector 2-front) away from the centre of the panel, the overall weight ( $80 \mathrm{~kg}$ per enclosure) and projection capability had to be considered. The chosen projectors weigh approximately $8 \mathrm{~kg}$ (including lens) each, having

\footnotetext{
${ }^{3}$ Point Grey Grasshopper 2 GigE.

${ }^{4} \mathrm{AV}-950 \mathrm{~T} / \mathrm{R}$.
}

5000 lumens brightness capability and can be programmed remotely from within the SAR software framework. At the time of the prototype design, more powerful projectors were not in consideration as they were significantly heavier. Fixed focus cameras were used for monitoring purposes. KVM switches were used to convert HDMI data stream to network data stream and vice-versa. Industrial-based gigabit network switches were used to cope with high data throughput and with environmental conditions (mainly heat) within the plant.

Apart from the network switches and cameras, all other equipment did not meet the plant criteria for installation onsite. Therefore, enclosures were designed with the appropriate material that conforms to plant regulations to protect the SAR equipment. The prototype enclosure is shown in Fig. 4.

The enclosures were engineered to withstand harsh environmental conditions (such as electromagnetic interference, vibration, heat, grease and weld sparks) within the manufacturing context, yet simultaneously provide a stable platform for the SAR system to operate from. Adequate cooling system was built into the compact design for long lasting operations. The enclosures were made from durable mild steel with powder coat finish. The enclosures have hinged front and rear doors with double bit spanner locks. There is a clear Perspex window at the front for projector and camera. Mounting rails and fixing holes were fitted in the inside corners of the enclosure. A power rail was fitted within the enclosure to provide power for all equipment inside the enclosure. The enclosure can be swivelled left to right (up to $60^{\circ}$ ) and can be rotated up/down (up to $60^{\circ}$ ). The enclosure is clamped to the I-beam with U-clamps. Loosening the clamps allows the enclosure to be moved up and down as required. The top of the enclosure is fitted with a hose for power and ethernet cables and eyelets for safety harness.

The robustness of the hardware was measured in terms of air temperature within the enclosures, whilst the SAR

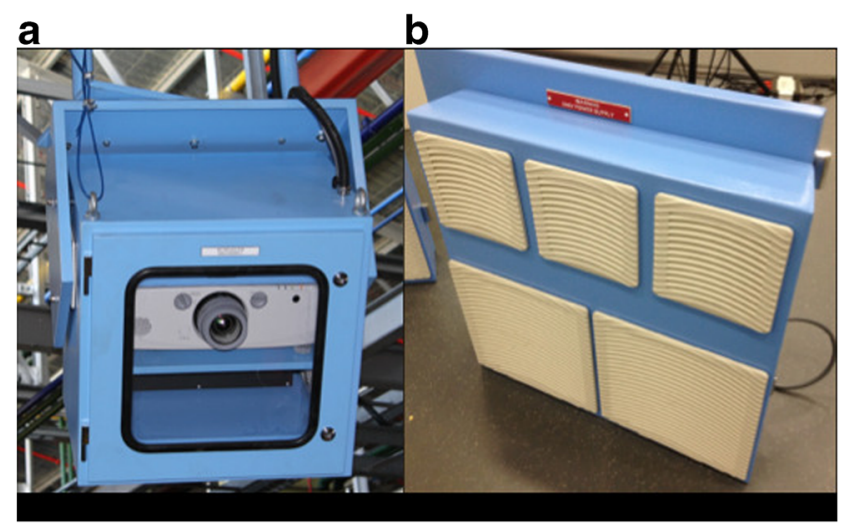

Fig. 4 SAR Prototype: a front view; $b$ rear view 


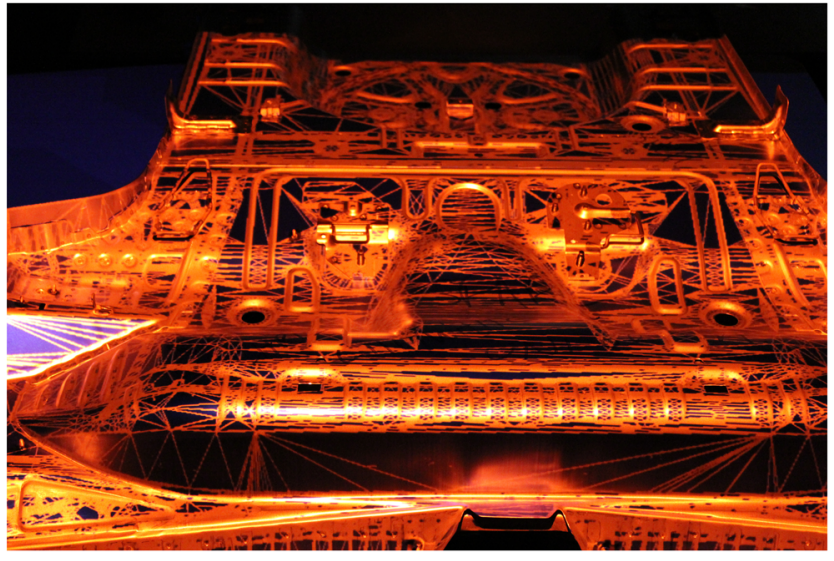

Fig. 5 After mapping 3D world points to 2D projection plane points, the engineering blueprint (wireframe of the $3 \mathrm{D}$ model) of the vehicle panel is projected onto the surface of the panel at the correct scale

system was operating continually. Over a two and a half week duration of Australian Winter (between July and August), the minimum temperature recorded was $13{ }^{\circ} \mathrm{C}$ and maximum was $33.5{ }^{\circ} \mathrm{C}$. The temperatures were also measured over 3 weeks during the Australian Summer (between December and January). The minimum air temperature recorded was $17.5^{\circ} \mathrm{C}$ and the maximum was $44^{\circ} \mathrm{C}$. This showed that the prototype design was practical and kept SAR equipment working within the recommended operating conditions at all times.

\subsection{Augmenting virtual objects on the panel}

The data handling process is simplified by prior extraction of the spot-weld locations in the world coordinate system. Using our software, the spot-weld locations are extracted based on production station requirements (also based on spot-weld metadata) and is also dependent on vehicle type. The system will then project specified visual cues onto the vehicle surface at the extracted spot-weld locations. The projected locations will change depending on the current vehicle panel type on the fixture at the station and/or as dictated by manufacturing systems.

Projector calibration is performed by applying the method described in $[29,30]$, which maps 3D world points to $2 \mathrm{D}$ points on the projection plane. Each spot-weld's unit vector normal is estimated from their $3 \mathrm{D}$ location along with their corresponding rotational matrix. These values are then used in the visualisation process to project the visual cues in a projective view along the normal of the spot-weld. This means that whichever plane the spot-welds are located on, the cues will always be oriented correctly. Front and rear face culling is disabled during visual cues projection routine resulting in the virtual object always displayed on top of the mesh layer, to yield maximum operator visibility.

Once calibrated, prior to displaying the virtual cues, the mesh (obtained from engineering blueprint) of the panel is projected onto the panel. Since the panel itself is of neutral colour (galvanised iron), any synthesized texture or color can be projected to it making the surface more compelling or interactive. An example of the projected mesh onto the surface of the panel is shown in Fig. 5.

\subsection{Deployment}

The deployment of the SAR prototype was successful within plant operating conditions. Figure 6 depicts the installed hardware at GM Holden Vehicle Operations in Elizabeth, Australia. The operators' workflow remained as per existing process. No change to their foot flow was necessary. The only visible difference is that the operators were quicker at moving the weld guns to the correct location.

\section{Visualisation design}

Prior to the commissioning of the SAR prototype, the viability of using virtual aids was investigated in a laboratory environment. The tests were conducted at the Mawson
Fig. 6 SAR prototype at GM HVO, Elizabeth, Australia

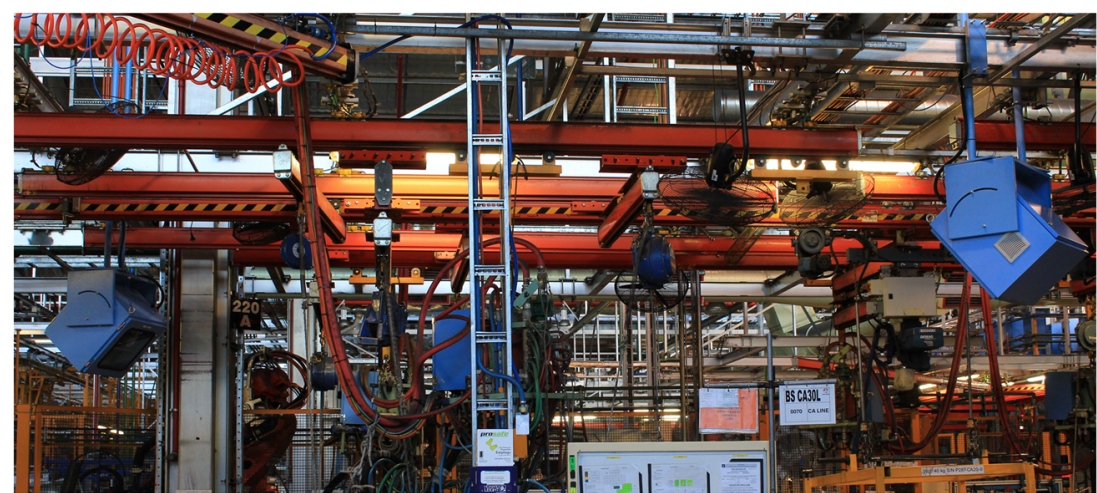


Institute Visualisation Lab (Holodeck), ${ }^{5}$ University of South Australia, Adelaide, Australia. The setup with a panel was laid out as depicted in Fig. 2. This simulated the layout of the station and positioning of the SAR prototypes with reference to the panel in the plant.

Designs and colours used for the visual cues were chosen for their simplicity (fast generation at $100 \mathrm{fps}$ ) and due diligence was made with respect to the welding operators on the production line. The 3D world coordinate locations of the spot-welds were extracted from the engineering blueprints for visual cues to be mapped accurately onto the panel. With the projector lenses approximately $4.5 \mathrm{~m}$ away from the centre of the panel, maximising the visual cue visibility for operators and maintaining virtual object sharpness is inherently important.

The primary colours for projective light (red, green and blue), yellow and white were initially used to test projections onto the surface of the vehicle panel. Whilst red, green, yellow and white were well lit and visible on the panel, the colour blue visibly stands out when it is used with another colour. The perception as a single color appears to be absorbed by the dull grey background of the metal surface. Other colours such as amber, purple, magenta, cyan, pink and orange were tested and rejected. Though they were visible when a large surface area was lit with the corresponding colours, they too were absorbed by the grey metal when only a single colored circle was projected to the spot-weld locations. Though colour blindness affecting operators was considered, we determined that a separate study of colour blindness for AR in manufacturing would be required, which was beyond the scope of this work. ${ }^{6}$ Hence, the colours used for the visual cues were determined to be appropriate.

Table 1 presents nine types of visual cues with varying colours (red, green, yellow, white and blue) and sizes. The table highlights the different designs used, corresponding design images and their projective examples on the panel. A short description for each type is also included in Table 1. The design motivation was to explore the extremes of the virtual aid requirement, i.e. from minimalistic to visually complex designs.

Following an expert review [32] performed by GM Holden Vehicle Operations, the minimalistic circle design with $15 \mathrm{~mm}$ radial circumference was chosen to be most suitable for the operators. The other designs were ineffective for the following reasons:

\footnotetext{
${ }^{5}$ http://www.unisa.edu.au/IT-Engineering-and-the-Environment/ Blog/augmenting-your-view-of-the-world1.

${ }^{6}$ Additional discussion on color blindness can be found in [31] which also reports that most people that suffer colour blindness is of the redgreen dichromat type. Byrne and Hilbert [31] also report that most sufferers of these type perceptually recognise red or green as either yellowish or blueish colours.
}

- Multiple circles (one within the other) could cause confusion to the operators.

- Crosses were acceptable but can cause significant confusion when spot-weld positions are too close to each other.

- The white circle with coloured crosses can be distracting to the operators.

- The large crosses with normals was too extreme and not advisable for production line. It overcrowded the surface area with colours and was distracting.

- Textured visual cues had too much colour, distracting and confusing especially when overlapped with other spot-welds. In addition, it was difficult to confirm whether the spot-weld at a location was welded or not.

Therefore, a simple and minimalistic design for a visual cue is best practise for use on the production line. However, during production trials, it was observed (visual inspection) that operators were welding within the tolerance outlined by the virtual aid but not towards the centre of the circle, which is the exact location of the spot-weld from the panel blueprints. Hence, the design from Table 1(i), circle with centre bullseye, was introduced to help the operators focus their weld guns towards the correct location. As will be shown in Section 7, this significantly improved the accuracy and precision of the manual spot-welders.

\section{Pilot study}

Before testing the system during production, an offline pilot study was conducted at the plant with an inexperienced operator. This work was not part of his daily routine. A completed panel (from the earlier shift) was kept on the fixture for the operator to perform a mock welding on the panel. This is so that the operator could familiarise themself with handling of the welding guns. Although the weld gun was fired, no weld was produced because the constant-current welding power supply was switched off. The existing panel was then hoisted away from the fixture towards the automated station and an incomplete panel was hoisted onto the fixture at this station. With the hoist moved aside and the clamps set on the panel, the SAR projection system was switched on to display the visual cues for the corresponding panel variant. The operator duly proceeded to perform his weld tasks for a set of spot and stud welds for this work station. Figure 7 depicts the first time the SAR system was used by an operator on the production line to spot-weld.

During the familiarisation process, the operator was struggling to place the weld gun at the welded positions and it took over $150 \mathrm{~s}$ to complete the task. With the virtual aid, the operator was able to complete the spot-welding task on the incomplete panel in just over $120 \mathrm{~s}$. This represented a 
Table 1 Types of visual cues

\begin{tabular}{|c|c|c|c|c|}
\hline ID Type & Visual Cue & Cue on Panel & Colours & Description \\
\hline (a) circle & & & green, yellow, red, white & $\begin{array}{l}\text { The design is simple, clear and not clut- } \\
\text { tered. The size can be adjusted to fit any } \\
\text { tolerance required. Different colours may } \\
\text { be applied for different modes of opera- } \\
\text { tion, i.e. panel variants and inspection. }\end{array}$ \\
\hline
\end{tabular}

(b) circle + inner circle
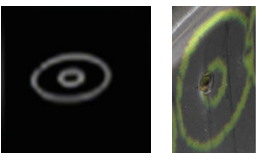

(c) cross + clear middle
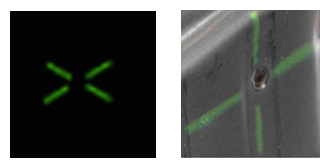

green, yellow, white

(d) white circle + coloured cross
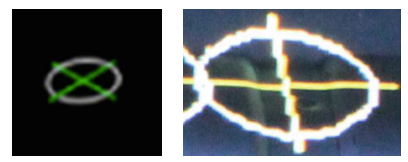

(e) cross
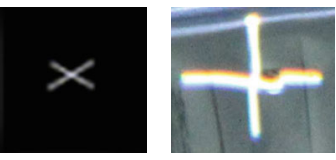

(f) large cross + normal
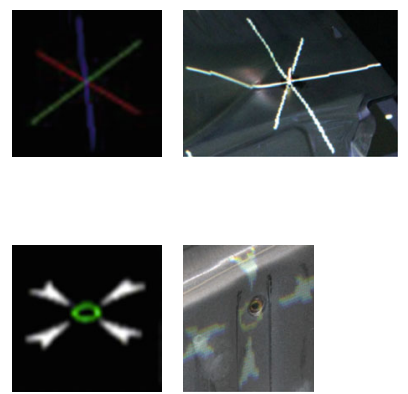

green, yellow, red

(g) white arrows + inner circle
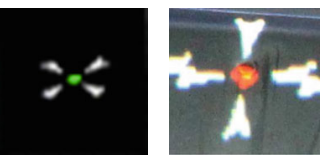

(i) circle + bullseye
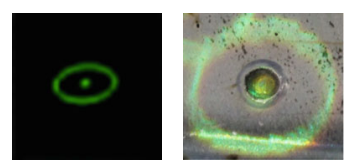

green, yellow, red, white

green, red, white, yellow, green+red+blue

green, yellow, red, white

green, yellow, red+blue, white

The outer circle highlights the welding region and the inner circle points to where the spot-weld should be placed. The spotweld fits in the inner circle so it is apparent whether welding has been performed or not.

The crosshair gives a precise location to aim the weld gun. The clear centre indicates where to weld. If there is a missed spot-weld, the operator can quickly verify by looking at the centre.

The crosshair gives a precise location to aim the weld gun. The outer circle highlights the welding region. The coloured crosshairs is meant to help the operator focus where to weld.

The crosshair gives a precise location to aim the weld gun.

The crosshair gives a precise location to aim the weld gun. The large size helps the operator to focus when they are tired. The surface normal adds another detail to the visual cue, which is the direction the projector is facing.

The white arrows indicate the direction of the spot-weld location. The coloured inner circle highlights the centre of the spot-weld, which the operator can use to quickly verify whether an error has been made.

green, yellow, red, white

The white arrows in the form of a crosshair indicates the direction of the spot-weld location. The coloured bullseye in the centre would help the operator to focus the weld gun quickly.

green, yellow

Minimalistic design. Helps the operator to intuitively place the weld gun in the correct region. The bullseye in the centre helps the operator to focus quickly and intuitively towards it.
$20 \%$ performance increase on a single panel with an inexperienced operator. The visualisations helped the operator to quickly position the weld guns in the correct locations for the welds. Furthermore, a faint mark was left on the panel when the weld gun was fired. On closer inspection after task completion, these marks (representing the actual spotweld when switched on) were distributed towards the centre of the circles compared to the spot-welds on the completed panel which were distributed towards the edge of the circles, although these are all within the guidelines [3]. This meant 


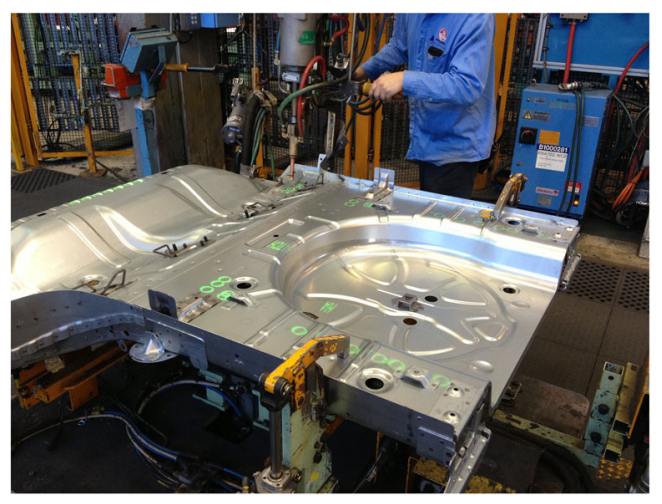

Fig. 7 Spot-welding during the pilot study

that the operator, despite his inexperience, was able to complete his welding task relatively quickly and more accurately with the SAR visualisations enabled.

\section{Production trials}

Following the successful test of the offline pilot study, the SAR visualisation system was trialled on a running production line with two variants of the GM Holden Cruze vehicles at the Elizabeth plant. The system was in use on four shifts between July, 2013 and October, 2013, to build roadworthy vehicles currently in use. This section presents our measurement methodology and production workflow to evaluate the performance of highly skilled automotive welders using the augmented reality system as a virtual aid. Comparative results between without-AR and with-AR assistance are presented and discussed later in this section.

\subsection{Validation methodology}

In order to compare the performance of manual spotwelding between without-AR and with-AR, quantifiable measurements such as accuracy and precision of the spotweld positions have to be taken. With the installed cameras approximately $4.5 \mathrm{~m}$ away from the centre of the panel and of fixed focus type to capture the whole panel, 66 spotand stud-welds distributed across a large surface area of the panel and the added restriction of no stoppages during the production cycle, it was a challenging task to devise a suitable method to obtain the measurements. To complicate the task further, the panels cannot be removed from the production line to retake measurements because they are being used to build motor vehicles. Therefore, only one opportunity exists to take measurements per panel.

To overcome these challenges, the measurement was constrained to six spot-welds which are at least $20 \mathrm{~mm}$ apart but clustered in a small group. A coloured radial chart (similar to that proposed in [7]) on a transparent acetate was made to obtain the measurements accurately. Acetate was considered because it is inexpensive and is able to withstand the heat of the spot-welds. The radial chart has $1 \mathrm{~mm}$ radial distance of concentric circles on the cluster of six spot-welds and the results were measured numerically for without- $A R$ and with-AR projections on the panel during production.

The measurement template used is shown in Fig. 8. It is an example with the template placed onto the panel after it was welded with AR assistance. The template is the exact scale as per the panel blueprints for accurate measurements. W1 to W6 are weld identification for the six spot-welds of interest on the panel. These spot-welds are identical for both variants of the GM Holden Cruze panels. The rings are up to $15 \mathrm{~mm}$ radial distance with $1-\mathrm{mm}$ intervals. This made for easy identification of the centre of the welds as defined by the design blueprints (marked as crosshairs on the template). Template calibration is performed by placing the hole punched $10 \mathrm{~mm}$ black ring on the far right over the stud-weld on the panel. Though the stud-welds are $6 \mathrm{~mm}$ in diameter, the additional room allowed for adjustments if required. The two black rings in the middle are datum hole positions on the panel. This made manual alignment easier between the template and panel.

\subsection{Evaluation procedure}

A total of eight trained operators across four shifts were involved in the trials. The operators followed the same routine as identified in the workflow in Section 3 for both without-AR and with-AR trials. Once the panel is secured and clamped onto the fixture, the SAR system augments visual cues to the surface of the panel. The operators proceed to weld using the guidance of the virtual aid. After welding is performed and visual checks completed, the panel is unclamped and hoisted away towards the rotating fixture. With the hoisting mechanism moved aside, the panel

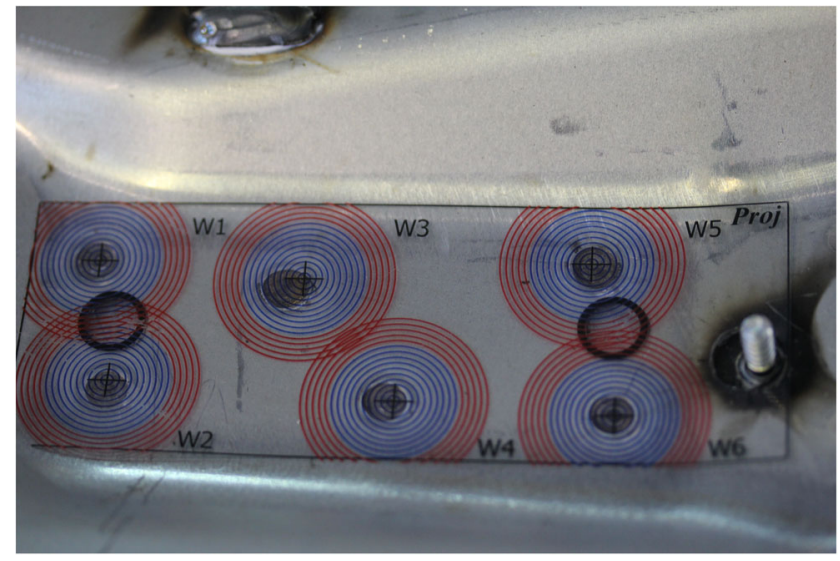

Fig. 8 Measurement template on the vehicle panel after welded with AR projections 
remains on the manual side of the fixture for approximately $30 \mathrm{~s}$ before it is rotated into the automated station. During those $30 \mathrm{~s}$, the template (radial chart) is placed onto the panel, a photograph is taken and template is removed. Template placement is done on the rotating fixture due to safety precautions, i.e high temperatures of spot-welds and to allow for the welds to be sufficiently cooled for the acetate to be placed on the surface. This ensured continuity during the production cycle with no operational impact.

\subsection{Performance evaluation}

With reference to Fig. 8, the coloured blue rings are up to $10 \mathrm{~mm}$ radial distance from its centre point the red rings are between 11 and $15 \mathrm{~mm}$ radial distance from its centre point.

Measurements were noted by identifying the centre of the spot-weld on the template first, then identifying which ring the spot-weld centre intersects with. Though not entirely exact (due to the application), it yields reasonable estimates for performance analysis. Figure 9 shows a spot-weld and its estimated centre point (red highlighted region).

The intersection point between the centre of the spotweld bead and the rings on the radial chart depicts the distance error $(d)$ of the welding relative to its ideal position as per the design blueprints. Hence, the error in accuracy (millimetres) per welded spot-weld is given by

$$
\begin{aligned}
\text { Accuracy Error } & =\text { Ideal Position }- \text { New Position } \\
& =d
\end{aligned}
$$

with mean error per spot-weld (W1-W6) calculated in millimetres as

Mean Error, $\bar{d}_{k}=\frac{\sum_{i=1}^{N} d_{k i}}{N}$

where $i$ is the spot-weld indices, $k$ is the spot-weld location (1-6 reflecting W1-W6 on the template) and $N$ is the

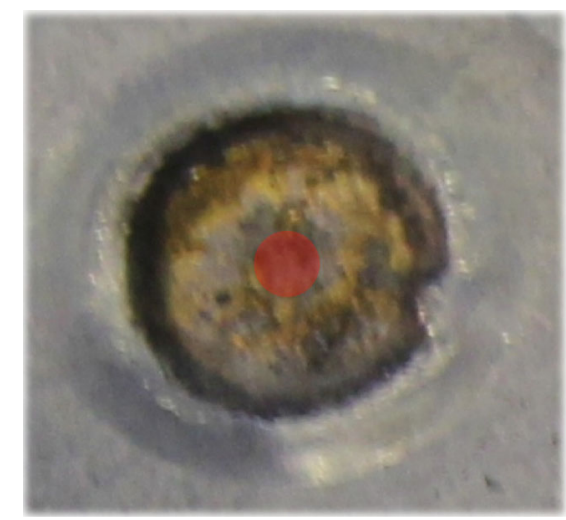

Fig. 9 Spot-weld with highlighted centre point total number of spot-welds (per location). The precision (the ability to repeatedly weld in the same location) for each spot-weld (W1-W6) is obtained by

Precision $=\sqrt{\frac{\sum_{i=1}^{N}\left(d_{k i}-\bar{d}_{k}\right)^{2}}{N-1}}$

Division is done with $N-1$ instead of $N$ because of the small sample size. The scatter plots presented in Figs. 10-11 are obtained by plotting the intersection point to both the $x$ - and $y$-axes.

\subsection{Results}

The production trials were conducted across four shifts at the GM Holden plant with a total of 476 vehicle panels observed in that time. Out of that total, 299 were welded without using AR visual cues and 177 were welded using AR visual cues. All panels were fitted into roadworthy vehicles currently in use. However, the measurement template was introduced towards the end of the trials. Hence, the performance figures reported are for 45 panels welded without using AR and 19 panels welded with AR assistance.

Figure 10 shows the welding pattern of without-AR and with-AR assisted spot-welds. These give a visual representation of the recorded measurements and provide distinct differences between without-AR and with-AR spot-weld positions. The blue band represents the $10 \mathrm{~mm}$ radial range and the red band represents the $11-20 \mathrm{~mm}$ radial range as per [3].

Figure 11 show the overall welding accuracy and precision for each spot-weld (W1-W6). Blue diamond in the figure depicts locations of spot-welds without-AR and red square are for with-AR spot-welds.

Independent $t$ tests were performed on the six weld positions comparing the position errors for the conditions of given projected augmented reality cues and not given projected augmented reality cues. The notations used for the statistical analysis are $M$ is mean, $S E$ is standard error of the mean, $C I$ is the confidence interval, $t(\cdot)$ is the $\mathrm{t}$ test value with the corresponding degree of freedom, $p$ is the statistical significance value, and $d_{c}$ is Cohen's effect size measure [33], which measures the mean difference between without-AR and with-AR data. The following are the statistical results obtained for each spot-weld from the $t$ tests.

W1 On average, a welder given the projected augmented reality cues performed welding with less radial error $(\mathrm{M}=$ $3.53 \mathrm{~mm}, \mathrm{SE}=0.27)$, than the welder not given the projected augmented reality cues $(\mathrm{M}=4.82 \mathrm{~mm}, \mathrm{SE}=0.42)$. This difference, $1.296 \mathrm{~mm}$, at $95 \%$ CI $0.263,2.270$, was 
Fig. 10 Welding pattern for a without-AR spot-welds and $\mathbf{b}$ with-AR spot-welds
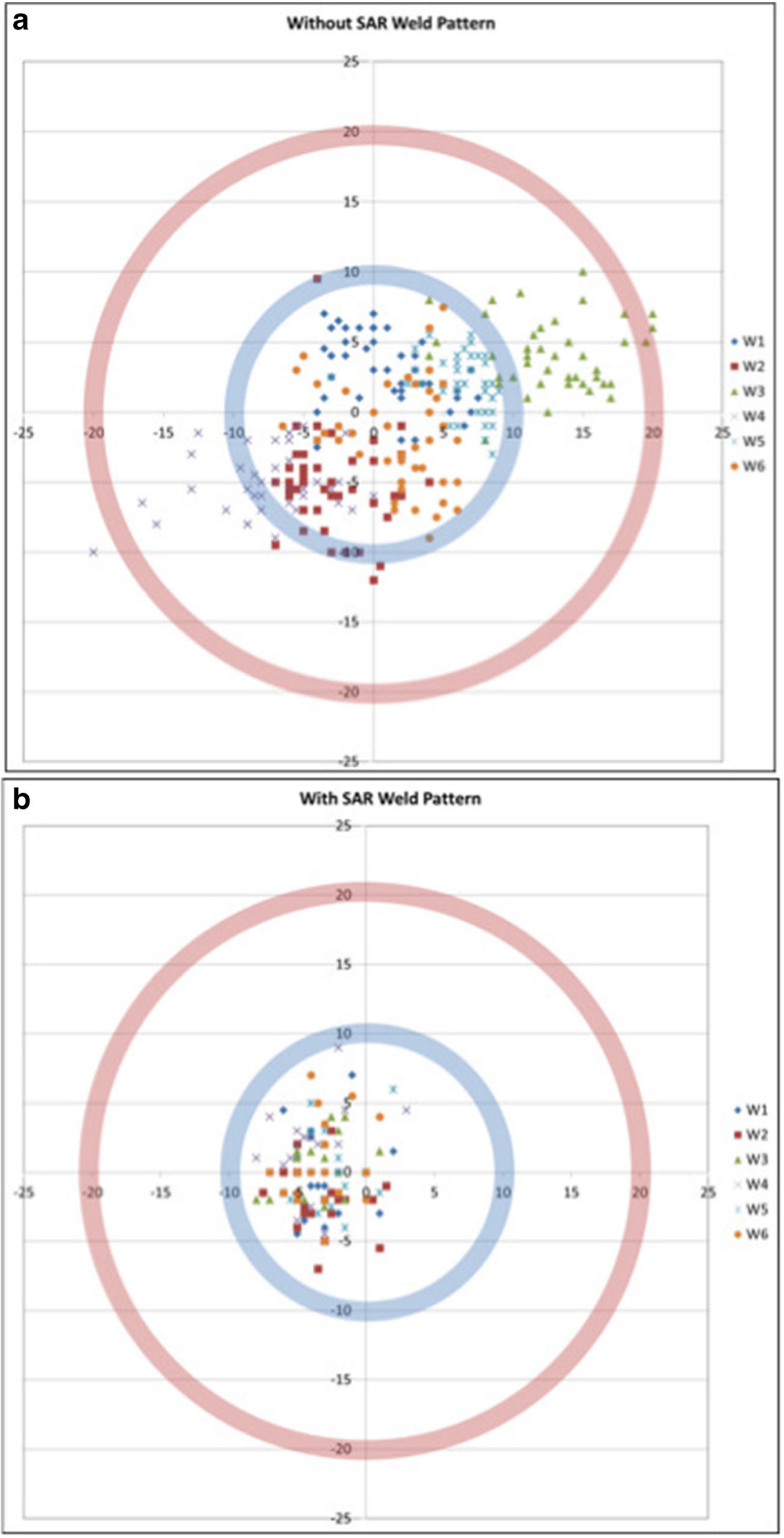

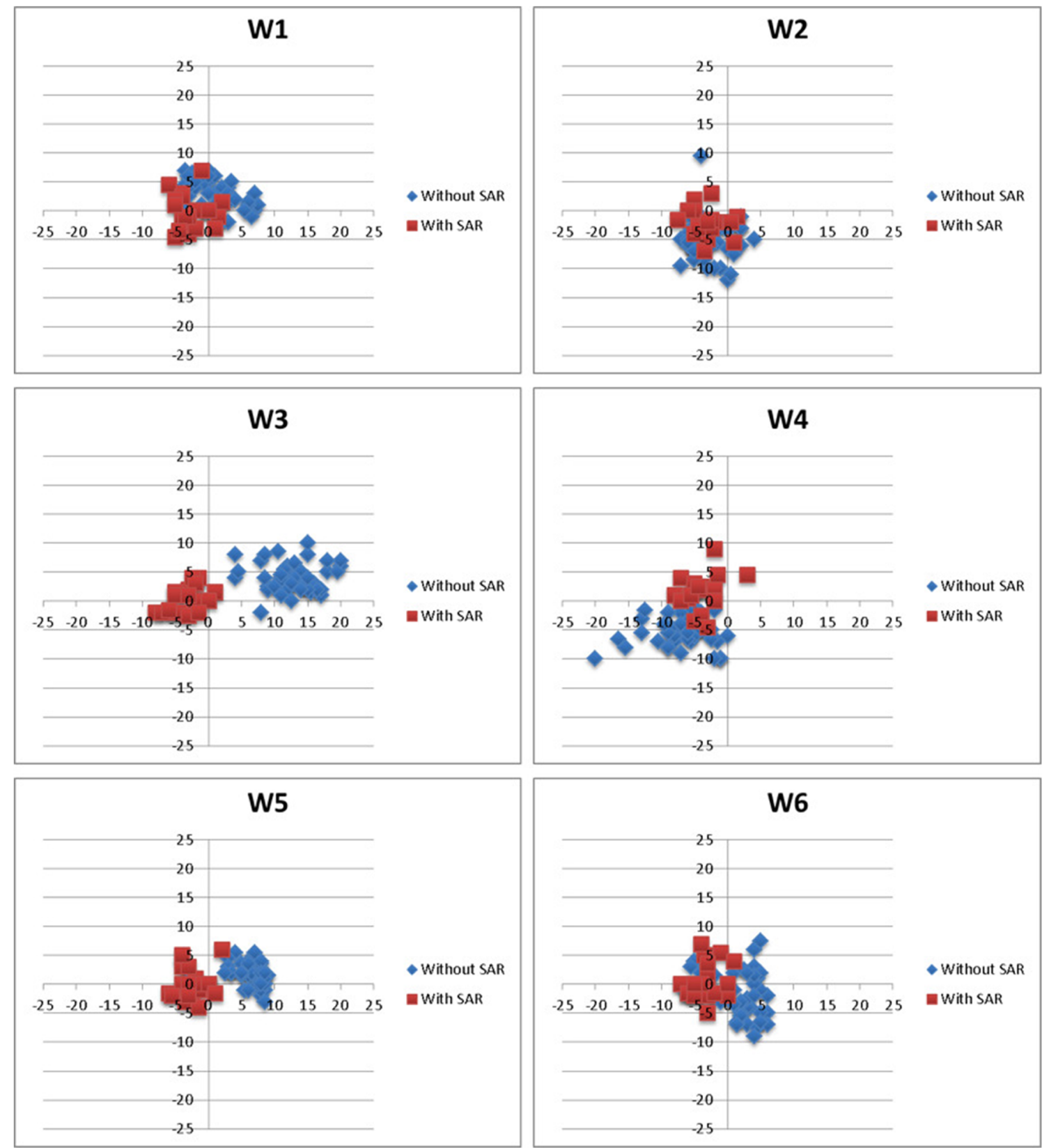

Fig. 11 Welding accuracy and precision for corresponding six spot-welds in the trial

significant, i.e. $t(62)=2.62, p=0.011$. This represented a medium-sized effect with $d_{c}=0.73$.

W2 On average, a welder given the projected augmented reality cues performed welding with less radial error (M $=4.42 \mathrm{~mm}, \mathrm{SE}=0.40$ ), than the welder not given the projected augmented reality cues $(\mathrm{M}=6.96 \mathrm{~mm}, \mathrm{SE}=0.37)$. This difference, $2.54 \mathrm{~mm}$, at $95 \%$ CI $1.219,3.850$, was significant, i.e. $t(62)=3.85, p<0.001$. This represented a large-sized effect with $d_{c}=0.97$.
W3 On average, a welder given the projected augmented reality cues performed welding with less radial error $(\mathrm{M}=$ $4.00 \mathrm{~mm}, \mathrm{SE}=0.44)$, than the welder not given the projected augmented reality cues $(\mathrm{M}=13.73 \mathrm{~mm}, \mathrm{SE}=0.57)$. This difference, $9.733 \mathrm{~mm}$, at $95 \%$ CI $8.303,11.164$, was significant, i.e. $t(59.807)=13.608, p<0.001$. This represented a large-sized effect with $d_{c}=2.57$.

W4 On average, a welder given the projected augmented reality cues performed welding with less radial error 
$(\mathrm{M}=5.32 \mathrm{~mm}, \mathrm{SE}=0.42)$, than the welder not given the projected augmented reality cues $(\mathrm{M}=9.16 \mathrm{~mm}, \mathrm{SE}=$ 0.56). This difference, $3.840 \mathrm{~mm}$, at $95 \%$ CI 2.452, 5.228, was significant, i.e. $t(60.375)=5.532, p<0.001$. This represented a large-sized effect with $d_{c}=1.03$.

W5 On average, a welder given the projected augmented reality cues performed welding with less radial error $(\mathrm{M}=$ $3.11 \mathrm{~mm}, \mathrm{SE}=0.41)$, than the welder not given the projected augmented reality cues $(\mathrm{M}=7.07 \mathrm{~mm}, \mathrm{SE}=0.26)$. This difference, $3.961 \mathrm{~mm}$, at $95 \%$ CI 3.008, 4.915, was significant, i.e. $t(62)=8.302, p<0.001$. This represented a large-sized effect with $d_{c}=2.33$.

W6 On average, a welder given the projected augmented reality cues performed welding with less radial error $(\mathrm{M}=$ $4.42 \mathrm{~mm}, \mathrm{SE}=0.45)$, than the welder not given the projected augmented reality cues $(\mathrm{M}=5.18 \mathrm{~mm}, \mathrm{SE}=0.34)$. This difference, $0.757 \mathrm{~mm}$, at $95 \%$ CI $-0.337,1.850$, was not significant, whereby $t(62)=1.276, p=0.170$. This represented a small-sized effect with $d_{c}=0.33$.

\subsection{Discussion}

With reference to Fig. 10a for non-assisted AR spot-welds, most welding is concentrated within or on the edge of the $10 \mathrm{~mm}$ radial range, relating to a small error distribution. However, most of the W3 and W4 spot-welds are within or on the edge of the $20 \mathrm{~mm}$ range which are acceptable as per the guidelines, but yields a higher error distribution. Freeform area of the surface, weld gun ergonomics and human error are some of the causes for W3 and W4 to have a higher error from the ideal locations. This highlights the reduced precision of manual spot-welding without guidance. W1, $\mathrm{W} 2$, W5 and W6 have tighter distribution within the $10 \mathrm{~mm}$ range, but this is attributed to the placement of the weld gun in the four corners of the panel underside. Please note that there is an acceptable $+/-2 \mathrm{~mm}$ error (due to $x$ and $y$ coordinate extraction from the images) included in the weld pattern visualisations.

In contrast and with reference to Fig. 10b, this highlights that using AR visual cues have improved the welding distribution to within the $10 \mathrm{~mm}$ radial range. This helped in achieving approximately $71 \%$ better accuracy (compared to non-AR assisted) for $\mathrm{W} 3$ and $42 \%$ (compared to non-AR assisted) for W4. There were also significant improvements for the W1 (27\%), W2 (36\%) and W5 (56\%) spot-welds, with W6 achieving a reasonable $15 \%$ improvement.

As observed in Fig. 11, the accuracy of manual spotwelding with-AR is better compared to without-AR, especially for W1, W3 and W5. The cluster of weld points for with-AR suggests that precision (repeatability of welding in the same location) is much better for W3, W4 and
W6, with W1, W2 and W5 having similar precision levels for both sets. The precision for all six spot-weld positions welded without-AR is $4.08 \mathrm{~mm}$ whilst with-AR, the precision is higher at $1.94 \mathrm{~mm}$. This accounts to approximately $52 \%$ increase in precision for trained operators with a significantly smaller error distribution.

\section{Conclusions}

Results show that there are gains to be made towards accuracy and precision using the SAR projection system compared to current spot-welding practices. Although there were improvements of at least $15 \%$ (W1, W2, W5 and W6) with weld gun having physical constraints, the largest improvement was in the free form regions of the panel (W3 and W4). The results are further validated statistically by confirming that there were small to medium improvements for W1 and W6 whilst large improvements were made for W2, W3, W4 and W5. This enforces our hypothesis that using augmented visual cues yields spot-welds that are closer to the optimal design location with higher accuracy and precision. This demonstrates the benefit of having an AR projection system on the production line as opposed to not having one. This work has also introduced an enhanced mechanism for quality assurance, which can be used for localised inspection for every panel without affecting production cycle and operators. The visual cues helped to identify spot-weld locations quicker and has the potential to be used as a training tool for new operators or for new vehicle types. This ensures continuous assurance for every vehicle. The SAR projection system can be positioned at any manual production station for spot welding, welding, adhesive application and inspection.

Open Access This article is distributed under the terms of the Creative Commons Attribution 4.0 International License (http:// creativecommons.org/licenses/by/4.0/), which permits unrestricted use, distribution, and reproduction in any medium, provided you give appropriate credit to the original author(s) and the source, provide a link to the Creative Commons license, and indicate if changes were made.

Acknowledgements The authors would like to thank University of South Australia, AutoCRC, GM Holden Vehicle Operations, GM Holden Innovations and GM International in support of this work.

\section{References}

1. Spot Track., http://www.spottrack.eu/welcome/standards-testing. jsp

2. Denisov A, Shakarji C, Lawford B, Maev R, Paille J (2004) Spot weld analysis with 2D ultrasonic arrays. J Res Natl Inst Stand Technol 109(2):233-244 
3. GM Worldwide Engineering Standards (2012) Weld Acceptance Criteria and Repair Procedures: Resistance Spot Welds - Steel. General Motors Worldwide, GMW14057

4. Lertrusdachakul I, Mathieu A, Aubreton O (2015) Vision-based control of wire extension in GMA welding. Int $\mathrm{J}$ Adv Manuf Technol:1-10

5. White S, Prachyabrued M, Baghi D, Aglawe A, Reiners D, Borst C, Chambers T (2009) Virtual welder trainer. In: Virtual Reality Conference, 2009, VR 2009. IEEE, pp 303-303

6. National Occupational Health and Safety Commission (1994) National Standard for Plant. Australian Publishing Service. NOHSC: 1010

7. Zhang H, Hou Y, Zhang J, Qi X, Wang F (2014) A new method for nondestructive quality evaluation of the resistance spot welding based on the radar chart method and the decision tree classifier. Int J Adv Manuf Technol:1-11

8. Lin HL, Chou T, Chou CP (2007) Optimization of resistance spot welding process using taguchi method and a neural network. Exp Tech 31(5):30-36. doi:10.1111/j.1747-1567.2007.00186.x

9. Xu W, Lin S, Fan C, Yang C (2015) Prediction and optimization of weld bead geometry in oscillating arc narrow gap all-position GMA welding. Int $\mathrm{J}$ Adv Manuf Technol:1-14. doi:10.1007/s00170-015-6818-7

10. Liu W, Ma J, Liu S, Kovacevic R (2015) Experimental and numerical investigation of laser hot wire welding. Int $\mathrm{J}$ Adv Manuf Technol:1-15

11. Ryuh BS, Pennock GR (2006) Arc welding robot automation systems. In: Huat LK (ed) Industrial Robotics: Programming, Simulation and Applications, InTech

12. Campanelli LC, Suhuddin UFH, İtalo Sette Antonialli A, dos Santos JF, de Alcántara NG, Bolfarini C (2013) Metallurgy and mechanical performance of AZ31 magnesium alloy friction spot welds. J Mater Process Technol 213(4):515-521

13. Chandrasekhar N, Vasudevan M, Bhaduri A, Jayakumar T (2015) Intelligent modeling for estimating weld bead width and depth of penetration from infra-red thermal images of the weld pool. J Intell Manuf 26(1):59-71. doi:10.1007/s10845-013-0762-x

14. Echtler F, Sturm F, Kindermann K, Klinker G, Stilla J, Trilk J, Najafi $H$ (2003) The intelligent welding gun: augmented reality for experimental vehicle construction. In: Virtual and Augmented Reality Applications in Manufacturing, pp 333360

15. Pentenrieder K, Bade C, Doil F, Meier P (2007) Augmented reality-based factory planning - an application tailored to industrial needs. In: The 6th IEEE International Symposium on Mixed and Augmented Reality, pp 31-42

16. Reiners D, Stricker D, Klinker G, Müller S (1999) Augmented reality for construction tasks: Doorlock assembly. In: Proceedings of the International Workshop on Augmented Reality : Placing Artificial Objects in Real Scenes: Placing Artificial Objects in Real Scenes, A. K. Peters, Ltd., IWAR '98, pp 31-46
17. Moehring M, Gloystein A, Doerner R (2009) Issues with virtual space perception within reaching distance: mitigating adverse effects on applications using HMDs in the automotive industry. In: Virtual Reality Conference, 2009, VR 2009, IEEE, pp 223226

18. Weidenhausen J, Knoepfle C, Stricker D (2003) Lessons learned on the way to industrial augmented reality applications, a retrospective on ARVIKA. Comput Graph 27(6):887-891

19. Regenbrecht H, Baratoff G, Wilke W (2005) Augmented reality projects in the automotive and aerospace industries. IEEE Comput Graph Appl 25(6):48-56

20. Reinhart G, Patron C (2003) Integrating augmented reality in the assembly domain - fundamentals, benefits and applications. CIRP Ann Manuf Technol 52(1):5-8

21. Pentenrieder K, Bade C, Doil F, Meier P (2007) Augmented reality-based factory planning - an application tailored to industrial needs. In: Mixed and Augmented Reality, 2007. ISMAR 2007. 6th IEEE and ACM International Symposium on, pp 31-42

22. Kollatsch C, Schumann M, Klimant P, Wittstock V, Putz M (2014) Mobile augmented reality based monitoring of assembly lines. Procedia CIRP 23(0):246-251. 5th CATS 2014 - CIRP Conference on Assembly Technologies and Systems

23. Servản J, Mas F, Menèndez JL, Rios J (2012) Using augmented reality in AIRBUS A400M shop floor assembly work instructions. AIP Conf Proc 1431(1):633-640. doi:10.1063/1.4707618

24. Zhou J, Lee I, Thomas B, Menassa R, Farrant A, Sansome A (2012) In situ support for automotive manufacturing using spatial augmented reality. Inter J Virtual Reality 11(1):33-41

25. Victoria Worksafe Australia (2007) Occupational Health and Safety Regulations. Worksafe Victoria, Part 3. 5 (Plant)

26. Advanced Realtime Tracking., http://www.ar-tracking.com/home/

27. Schwerdtfeger B, Pustka D, Hofhauser A, Klinker G (2008) Using laser projectors for augmented reality. In: Proceedings of ACM Symposium on Virtual Reality Software and Technology, pp 134137

28. Neumann U, Majoros A (1998) Cognitive, performance, and systems issues for augmented reality applications in manufacturing and maintenance. In: Virtual Reality Annual International Symposium, 1998. Proceedings., IEEE 1998, pp 4-11

29. Raskar R, Brown M, Yang R, Chen WC, Welch G, Towles H, Scales B, Fuchs H (1999) Multi-projector displays using camerabased registration. In: Visualization '99. Proceedings, pp 161522

30. Piper B, Ishii H (2001) CADcast: a method for projecting spatially referenced procedural instructions. Tech. rep., MIT Media Lab

31. Byrne A, Hilbert D (2010) How do things look to the color-blind In: Color Ontology and Color Science, The MIT Press

32. Lazar J, Feng JH, Hochheiser H (2010) Research Methods in Human-Computer Interaction. Wiley Publishing

33. Cohen Jacob (1998) Statistical Power Analysis for the Behavioral Sciences, 2nd edn. Lawrence Erlbaum Associates, Publishers 